\title{
Probiotic Effects of Lactobacillus fermentum ZJUIDS06 and Lactobacillus plantarum ZY08 on Hypercholesteremic Golden Hamsters
}

\author{
Dongting Yang ${ }^{1+}$, Wentao $\mathrm{Lyu}^{2 t}$, Ziyi Hu ${ }^{1+}$, Jiting Gao ${ }^{1}$, Zhiyao Zheng ${ }^{1}$, Weijun Wang ${ }^{3}$, \\ Jenni Firrman ${ }^{4}$ and Daxi Ren ${ }^{\text {t* }}$ \\ ${ }^{1}$ College of Animal Sciences, Institute of Dairy Science, Zhejiang University, Hangzhou, China, ${ }^{2}$ State Key Laboratory for \\ Managing Biotic and Chemical Threats to the Quality and Safety of Agro-Products, Institute of Agro-Product Safety and \\ Nutrition, Zhejiang Academy of Agricultural Sciences, Hangzhou, China, ${ }^{3}$ Zhejiang Yiming Food Co. Ltd., Wenzhou, China, \\ ${ }^{4}$ Dairy and Functional Foods Research Unit, Eastern Regional Research Center, Agricultural Research Service, U.S. \\ Department of Agriculture, Wyndmoor, PA, United States
}

OPEN ACCESS

Edited by:

Jie Yin,

Hunan Agricultural University, China

Reviewed by:

Long Zhang,

China West Normal University, China

Wei Zhang,

Beijing Academy of Agricultural and

Forestry Sciences, China

*Correspondence:

Daxi Ren

dxren@zju.edu.cn

†These authors have contributed equally to this work

Specialty section

This article was submitted to Nutrition and Microbes,

a section of the journal

Frontiers in Nutrition

Received: 06 May 2021

Accepted: 27 May 2021

Published: 28 June 2021

Citation:

Yang D, Lyu W, Hu Z, Gao J, Zheng Z,

Wang W, Firrman J and Ren D (2021)

Probiotic Effects of Lactobacillus

fermentum ZJUIDSO6 and

Lactobacillus plantarum ZY08 on

Hypercholesteremic Golden

Hamsters. Front. Nutr. 8:705763.

doi: 10.3389/fnut.2021.705763
Hypercholesteremia or high cholesterol is one of the important factors leading to atherosclerosis and other cardiovascular diseases. The application of probiotics with cholesterol-lowering characteristics has become increasingly popular over the past decade due to their contribution to human health. This study aimed to evaluate the probiotic effects of Lactobacillus fermentum ZJUIDSO6 and Lactobacillus plantarum ZY08 on hyperlipidemic golden hamsters. A hyperlipidemic model was established through a high cholesterol diet in golden hamsters, after which lyophilized Lactobacillus fermentum ZJUIDS06 and Lactobacillus plantarum ZY08 were orally administered individually for 8 weeks. The physiological characteristics of golden hamsters and short chain fatty acid (SCFA) in the colon were assessed by automatic Biochemical Analyzer and gas choromatograph, respectively. A MiSeq sequencing-based analysis of the bacterial 16S rRNA gene (V3-V4 region) in the cecum content was performed to analyze the cecum microbiota. Correlations between sets of these variables were also investigated using the R package "corrplot." Results showed that neither Lactobacillus fermentum ZJUIDS06 nor Lactobacillus plantarum ZY08 inhibited body weight increase. However, supplementation with Lactobacillus fermentum ZJUIDS06 for 8 weeks increased colon SCFA levels $(P<0.05)$, decreased serum low-density lipoprotein, total cholesterol, and triglycerides levels, and also induced changes in the cecum microbiota of hyperlipidemic golden hamsters. Remarkably, oral administration of Lactobacillus fermentum ZJUIDS06 increased the relative abundance of Parabacteroides in the cecum, which served as a biomarker for colon SCFA production and improvement of serum cholesterol levels. In a word, Lactobacillus fermentum ZJUIDS06 improved hyperlipidemia in golden hamsters, which correlated with an increase in SCFA levels and relative abundance of Parabacteroides, indicating its potential importance in functional foods that can help lower cholesterol.

Keywords: in vivo, probiotic potential, Lactobacillus fermentum, Lactobacillus plantarum, cholesterol-lowering effects, intestinal microbiota 


\section{INTRODUCTION}

Atherosclerosis is a type of cardiovascular disease that has become increasingly prevalent on a global scale and contributes to the etiology of multiple diseases, such as coronary heart disease, cerebral infarction, and peripheral vascular disease (1-3). The most commonly associated pathogenic factor for atherosclerosis is the increase in low-density lipoprotein cholesterol (LDL-C) levels in conjugation with a decrease in high-density lipoprotein cholesterol (HDL-C) levels $(4,5)$. The prescribed use of statin drugs reduces the risk of developing atherosclerotic cardiovascular disease $(6,7)$, cardiovascularrelated death, and death in general, by lowering LDL-C levels $(8-10)$. However, the use of statins on a regular basis has been associated with multiple adverse effects, including liver damage, liver necrosis, kidney damage, myopathy, and rhabodomyolysis $(6,11,12)$, asks for the development of alternative agents or bioactives with cholesterol-lowering characteristics.

The administration of cholesterol-lowering probiotics has become increasingly popular over the past few decades due to their generally recognized as safe (GRAS) status and their contribution to the healthy microbiota of human mucosal surfaces. To date, a number of cholesterol-lowering strains have been isolated from feces of healthy people, fermented dairy products, and pickles, including Lactobacillus plantarum, Lactobacillus fermentum, Lactobacillus acidophilus, Lactobacillus casei, Lactobacillus reuteri, Lactobacillus rhamnosus, Bifidobacterium, and Enterococcus faecium. The LAB strains previously reported to exhibit the cholesterollowering effect in vivo mainly belong to Lactobacillus and Enterococcus $(13,14)$.

The cholesterol-lowering effects of Lactobacillus fermentum or Lactobacillus plantarum have been reported from both animal models and human clinical trials. Lactobacillus fermentum MJM60397 reduced the levels of serum triglycerides (TG) and LDL-C, and improved gene expression of LDL-R in livers of male ICR mice after a 7-week intervention period (15). Consumption of buffalo milk fermented by Lactobacillus fermentum improved serum lipids and biochemical indexes of livers in male Wistar rats (16). Lactobacillus plantarum EM fermented juice reduced the levels of serum TG, total cholesterol (TC), and LDL-C of Sprague-Dawley rats and improved the expression of $7 \alpha$-hydroxylase and LDL receptors in the rat liver (17). In addition, Lactobacillus plantarum colonizing the colon of rats reduced serum alanine aminotransferase (ALT), aspartate aminotransferase (AST), TC, TG, LDL, very lowdensity lipoprotein (VLDL), and the Atherogenic Index under hypercholesterolemic conditions (18). In human clinical trials, ingestion of Lactobacillus fermentum ME-3 for 4 weeks decreased serum TG and oxidized-LDL and increased serum HDL-C, and thus reducing the risk of developing cardiovascular disease and diabetes (19). Heat-inactivated Lactobacillus plantarum L137 reduced the levels of serum TC, LDL-C, AST, and ALT in overweight people (20). In a human clinical trial, treatment with live Lactobacillus plantarum Q180 for 12 weeks decreased postprandial maximum concentrations of TG, LDL-C, Apo B100, and Apo B-48 levels (21).
Several mechanisms for cholesterol reduction by lactic acid bacteria (LAB) have been proposed, such as deconjugation of bile salts by bile-salt hydrolase (BSH) (22-24), binding and incorporation of cholesterol to the LAB cellular surface (2527), production of short-chain fatty acids (SCFAs) during the LAB growth $(28,29)$, and co-precipitation of cholesterol with deconjugated bile salts $(30,31)$. Nevertheless, the mechanism for cholesterol reduction by LAB needs to be studied on a case-tocase basis and the cholesterol-lowering effects of LAB still need to be elucidated.

In our previous study, two cholesterol-lowering probiotics, Lactobacillus plantarum ZY08 and Lactobacillus fermentum ZJUIDS06, were isolated from baby feces. Both strains demonstrated cholesterol-lowering effects in vitro (Supplementary Figure 1), were resistant to acid and bile salt, and had no antibiotic resistance. However, the in vivo cholesterollowering effects of these two strains were still unknown, and their effects on the intestine microbial community remained unclear. Therefore, the objectives of this study were to assess the effects of Lactobacillus plantarum ZY08 and Lactobacillus fermentum ZJUIDS06 on serum lipids, SCFA profiles, and gut microbiota in hyperlipidemic golden hamsters, and thus to provide deeper insights into the counter- hyperlipidemic effects of certain probiotics.

\section{MATERIALS AND METHODS}

\section{Bacterial Strains, Culture Conditions, and Gavage Administration}

Lactobacillus plantarum ZY08 and Lactobacillus fermentum ZJUIDS06 (Supplementary Figure 2) were isolated from breastfed baby (6 months old) feces in Hangzhou, Zhejiang Province, China. The two strains were grown in MRS broth (Beijing Land Bridge Technology Co. Ltd., Beijing, China) and incubated anaerobically at $37^{\circ} \mathrm{C}$ for $18 \mathrm{~h}$. In vitro cholesterol-lowering characteristics for the two strains (Supplementary Figure 1) were determined following previously described methods (32). The two strains were lyophilized (WECARE-BIO Biotechnology Co. Ltd., Jiangsu, China) and stored at $-20^{\circ} \mathrm{C}$ until use.

\section{Golden Hamster Experimental}

The in vivo experiment was conducted following a previous study with some modifications $(33,34)$. In this experiment, cholesterol $(0.1 \%)$ and lard (10\%) were added to the standard diet (Supplementary Table 1, Pluteng Biological Technology Co. Ltd., Shanghai, China) to produce the high cholesterol diet. Golden hamsters (Mesocricetus auratus, Vital River Laboratory Animal Technology Co. Ltd., Beijing, China) were fed the high cholesterol diet to develop the mixed hyperlipidemia model. Animal care and experimental procedures were approved prior to initiation (\#17426), and the guidelines set by the Animal Care and Use Committee of Zhejiang University was followed.

Golden hamsters were selected as the animal model for this study because hamsters synthesize and excrete cholesterol and bile acids in a manner similar to that of humans, 


\section{High cholesterol diet treatment: 6-week-old golden hamsters were fed with $0.1 \%$ cholesterol and $10 \%$ lard}

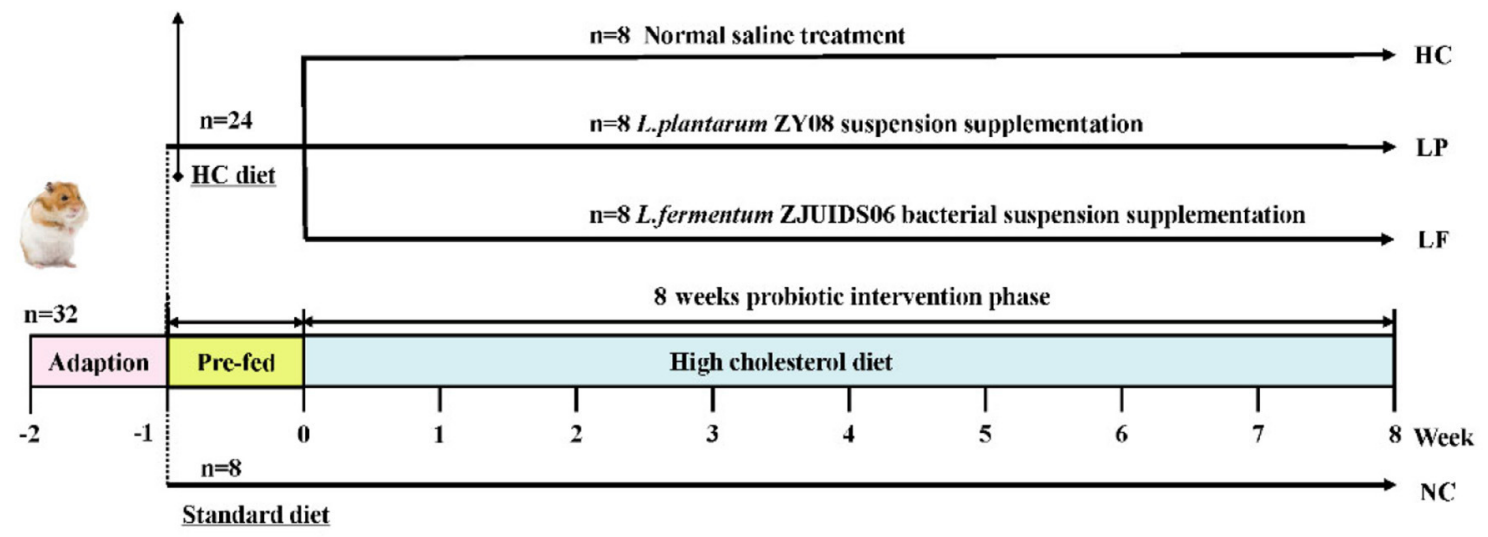

FIGURE 1 | Experimental design of animal study.

and they have become a standard model for evaluating the cholesterol-decreasing efficacy of probiotic strains (34-36). Male hamsters are considered a better model than females for developing hyperlipidemia and evaluating the cholesteroldecreasing efficacy because they are more susceptible to a highfat diet induced weight gain (37). Accordingly, male hamsters were chosen as a model to assess the cholesterol-lowering effect of LAB in vivo. A flow chart summarizing the aims of our study can be found in Figure 1. A total of 32 male golden hamsters, 6 weeks old, were fed a standard diet for 1 week to allow them to adapt to their new environment. Subsequently, 24 hamsters were fed the high cholesterol diet for 8 weeks, and the other eight hamsters were maintained using a standard diet for 8 weeks and were considered as the negative control group (NC group). To initiate the experimental phase, the 24 hamsters fed the high cholesterol diet were randomly assigned to the following three groups according to their body weight: High cholesterol positive control group (HC group), Lactobacillus plantarum ZY08 intervention group (LP group), and Lactobacillus fermentum ZJUIDS06 intervention group (LF group). During the experimental period, golden hamsters in the $\mathrm{NC}$ group and HC group were given $1 \mathrm{~mL}$ normal saline per $100 \mathrm{~g}$ body weight per day. Hamsters in the LP group were given $1 \mathrm{~mL}$ of Lactobacillus plantarum ZY08 suspension $\left(10^{9} \mathrm{CFU} / \mathrm{mL}\right)$ per $100 \mathrm{~g}$ body weight per day, while hamsters in the LF group were given $1 \mathrm{~mL}$ Lactobacillus fermentum ZJUIDS06 suspension $\left(10^{9}\right.$ $\mathrm{CFU} / \mathrm{mL}$ ) per $100 \mathrm{~g}$ body weight per day. The viable counts of lyophilized bacteria were enumerated on MRS agar by surface plating on a weekly basis. Lyophilized bacteria were suspended in saline and the viable cell counts in the suspension used for gavage administration were $\sim 10^{9} \mathrm{CFU} / \mathrm{mL}(35,38,39)$.

Body weight and food intake were measured every week and blood samples were collected every 2 weeks. After an 8-week intervention period, all golden hamsters were euthanized, and dissected before blood, liver, kidney, and intestine tract samples were collected and frozen $-80^{\circ} \mathrm{C}$ until analyzed.

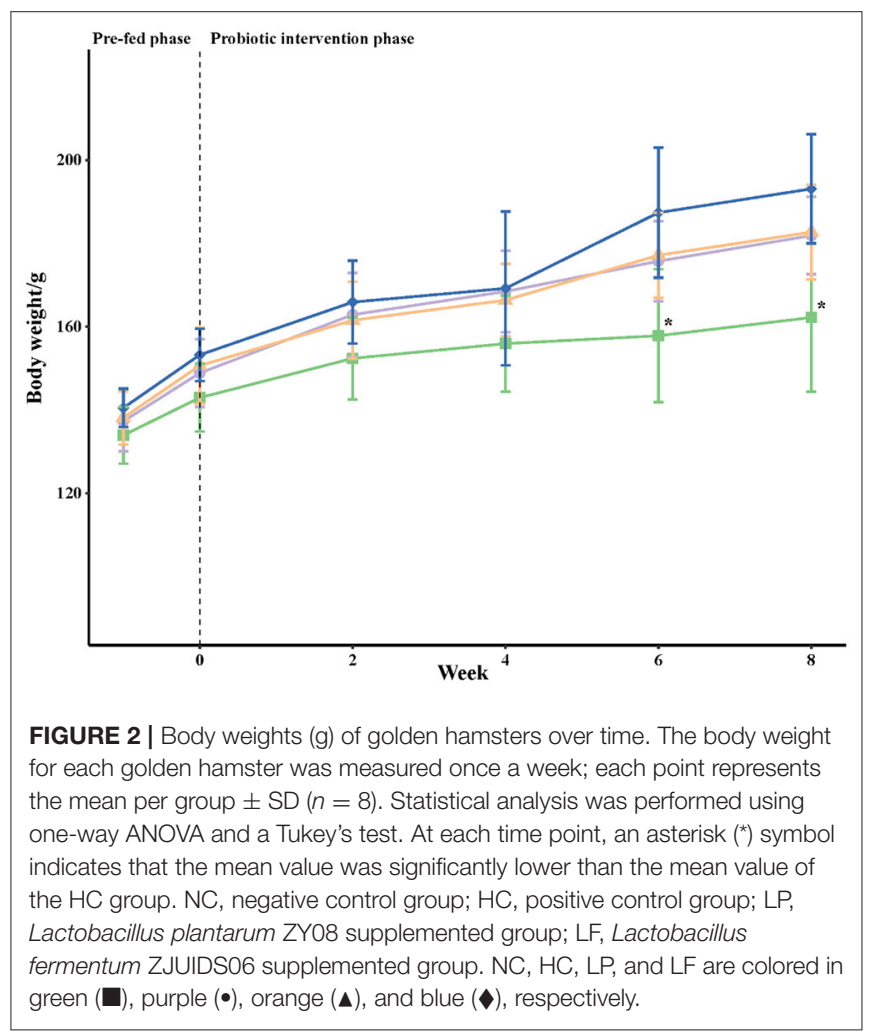

\section{Serum Lipids Determination}

Blood samples were collected every 2 weeks from the intraorbital venous plexus of the golden hamsters using a flat-ending capillary (0.5 mm diameter) tube. The blood was stored at $4^{\circ} \mathrm{C}$ overnight and centrifuged at $3000 \times \mathrm{g}$ for $10 \mathrm{~min}$ to harvest the serum for lipid profiling. The amounts of TC, LDL-C HDL-C, and TG in the serum were determined by automatic Biochemical 
Analyzer (\#3100, Hitachi, Ltd., Tokyo, Japan), according to the manufacturer's procedure.

\section{Histological Examination of the Liver}

The livers of the golden hamsters were frozen in liquid nitrogen immediately after dissection. The tissue was covered by an OCT embedding agent (Tissue-Tek O.C.T. Compound 4583, SAKURA Finetek USA, Torrance, USA), frozen, and then cut out using Cryotome E (Thermo Fisher Scientific, Waltham, USA). A coverslip (Servicebio Technology Co. Ltd., Wuhan, China) was applied to attach the tissue. Finally, the hematoxylin-eosin staining was performed, and the section was observed using an oil immersion lens (Eclipse E100, Nikon, Corp., Tokyo, Japan) and scanned by Pannoramic MIDI (3DHISTECH ${ }^{\mathrm{TM}}$ Ltd., $^{-}$ Budapest, Hungary).

\section{Determination of SCFA}

SCFA in the colon content were determined following a previously described method with some modification (40). After dissection, the segmented colon sections were squeezed with sterile forceps, and the contents were removed and stored in cryopreservation tubes at $-80^{\circ} \mathrm{C}$. The colon contents were diluted five-fold with ultrapure water and vortexed for $3 \mathrm{~min}$. Next, the suspension was rested for $5 \mathrm{~min}$ and then centrifuged at $4^{\circ} \mathrm{C}, 5000 \times \mathrm{g}$ for $20 \mathrm{~min}$. One milliliter of supernatant was mixed with $20 \mu \mathrm{L}$ chromatogram grade phosphoric acid (Shanghai Aladdin Biochemical Technology Co. Ltd.), and the mixture was injected into a chromatographic vial (WondaVial, Shimadzu, Corp., Kyoto, Japan) through a $0.45 \mu \mathrm{m}$ membrane filter for gas chromatography.

The gas chromatography machine (Shimadzu, Corp., Kyoto, Japan) consisted of an AOC-20S auto sampler and a GC-2010 equipped with a flame ionization detector. A SH-stabliwax (\#227$36305-2,30 \times 0.25 \times 0.25$, Shimadzu, Corp., Kyoto, Japan) highly polar column was installed on the GC with nitrogen as the carrier gas at a flow rate of $3 \mathrm{~mL} / \mathrm{min}$. The sample injection volume was $0.2 \mu \mathrm{L}$ with a split injection ratio of 50 and an injection temperature of $200^{\circ} \mathrm{C}$. The ethyl acetate was injected as a blank solvent between every sample to remove any memory effects. The initial column temperature was set at $80^{\circ} \mathrm{C}$ and held for $1 \mathrm{~min}$, then increased to $170^{\circ} \mathrm{C}$ at a rate of $8^{\circ} \mathrm{C} / \mathrm{min}$, then immediately increased to $220^{\circ} \mathrm{C}$ at a rate of $20^{\circ} \mathrm{C} / \mathrm{min}$ and maintained for $4 \mathrm{~min}$. The total time was $18.75 \mathrm{~min}$. Finally, the content of SCFAs was calculated according to the SCFA standard curve, which was calibrated by the external standard method.

\section{Tissue Weight}

At the end of the experiment, the adipose tissue surrounding liver, kidney, and epididymis were collected, washed with PBS, and dried with clean filter paper. The tissues were then weighed by using an electronic balance (BSA124, Sartorius, Inc., Gottingen, Germany) and recorded.

\section{DNA Extraction and Cecum Microbiota Analysis}

The cecum content usually contains the highest absolute number and diversity of microorganisms in the gastrointestinal tract (41), and is thus particularly useful for the analysis of microbiota. After dissection, the segmented cecum was squeezed with sterile forceps to remove the content, which was stored in cryopreservation tubes at $-80^{\circ} \mathrm{C}$. DNA from the cecum content was extracted using the Fast DNA SPIN extraction kit (MP Biomedicals, Inc., Santa Ana, USA) following the manufacturer's protocol. Quantity and quality of the extracted DNA were measured using the NanoDrop DN-1000 spectrophotometer (Thermo Fisher Scientific, Inc., Waltham, USA) and agarose gel electrophoresis, respectively. PCR amplification of the bacterial $16 \mathrm{~S}$ rRNA genes V3-V4 region was performed using the forward primer $338 \mathrm{~F}\left(5^{\prime}\right.$-ACTCCTACGGGAGGCAGCA-3 $\left.{ }^{\prime}\right)$ and the reverse primer 806R ( $5^{\prime}$-GGACTACHVGGGTWTCTAAT$3^{\prime}$ ) (Personal Biotechnology Co. Ltd., Shanghai, China) (42-44). Sample-specific 7-bp barcodes were added to the primers for multiplex sequencing. The PCR components consisted of $5 \mu \mathrm{L}$ of Q5 reaction buffer $(5 \times), 5 \mu \mathrm{L}$ of Q5 High-Fidelity GC buffer $(5 \times), 0.25 \mu \mathrm{L}$ of Q5 High-Fidelity DNA polymerase $(5 \mathrm{U} / \mu \mathrm{L}), 2$ $\mu \mathrm{L}(2.5 \mathrm{mmol} / \mathrm{L})$ of dNTPs, $1 \mu \mathrm{L}(10 \mu \mathrm{mol} / \mathrm{L})$ of each forward and reverse primer, $2 \mu \mathrm{L}$ of DNA template, and $8.75 \mu \mathrm{L}$ of $\mathrm{dd}_{2} \mathrm{O}$. Thermal cycling covered initial denaturation at $98^{\circ} \mathrm{C}$ for $2 \mathrm{~min}$, followed by 25 cycles including denaturation at $98^{\circ} \mathrm{C}$ for $15 \mathrm{~s}$, annealing at $50^{\circ} \mathrm{C}$ for $30 \mathrm{~s}$, and extension at $72^{\circ} \mathrm{C}$ for $30 \mathrm{~s}$, with a final extension of $5 \mathrm{~min}$ at $72^{\circ} \mathrm{C}$. The Agencourt AMPure Beads (Beckman Coulter, Inc., Indianapolis, USA) were applied for PCR amplicon purification and a PicoGreen dsDNA Assay Kit (Invitrogen, Thermo Fisher Scientific, Waltham, USA) was used for quantification. Based on quantification, amplicons were gathered in equal amounts, and pair-end $2 \times 300$ bp sequencing was performed using the Illlumina MiSeq platform with the MiSeq Reagent Kit v3 at Shanghai Personal Biotechnology Co. Ltd. (Shanghai, China). The sequencing data were uploaded to the Sequence Read Archive (SRA) of NCBI and can be viewed with the following accession code: PRJNA727412.

Bioinformatics were applied to the sequencing data using QIIME2 (45) with slight modifications. Briefly, raw sequence data were demultiplexed using the demux plugin and then the primer removed with the cut adapt plugin (46). Sequences were quality filtered, de-noised, merged, and chimeras were removed using the DADA2 plugin (47). Mafft (48) was applied to align the non-singleton amplicon sequence variants (ASVs) and then the results were applied to construct a phylogeny with fasttree2 (49). The diversity plugin was applied to estimate the Alpha-diversity metrics Chaol (50). The observed species, Shannon $(51,52)$, and Simpson (53), and the beta diversity metrics, weighted UniFrac (54), unweighted UniFrac (55), and Bray-Curtis dissimilarity were identified. All samples were rarefied to 56,522 sequences. The classify-sklearn naïve Bayes Taxonomy classifier in featureclassifier plugin (45) was applied to assign Taxonomy to ASVs set against the SILVA Release 132 Database (56).

\section{Statistical Analysis}

Experiments were performed in biological triplicates. All data are expressed as means \pm SD. Differences between variables were tested for significance by one-way ANOVA with a Tukey's Test (General parameter data) or a Kruskal-Wallis test (Nonparametric data) using IBM SPSS version 24.0 (International 

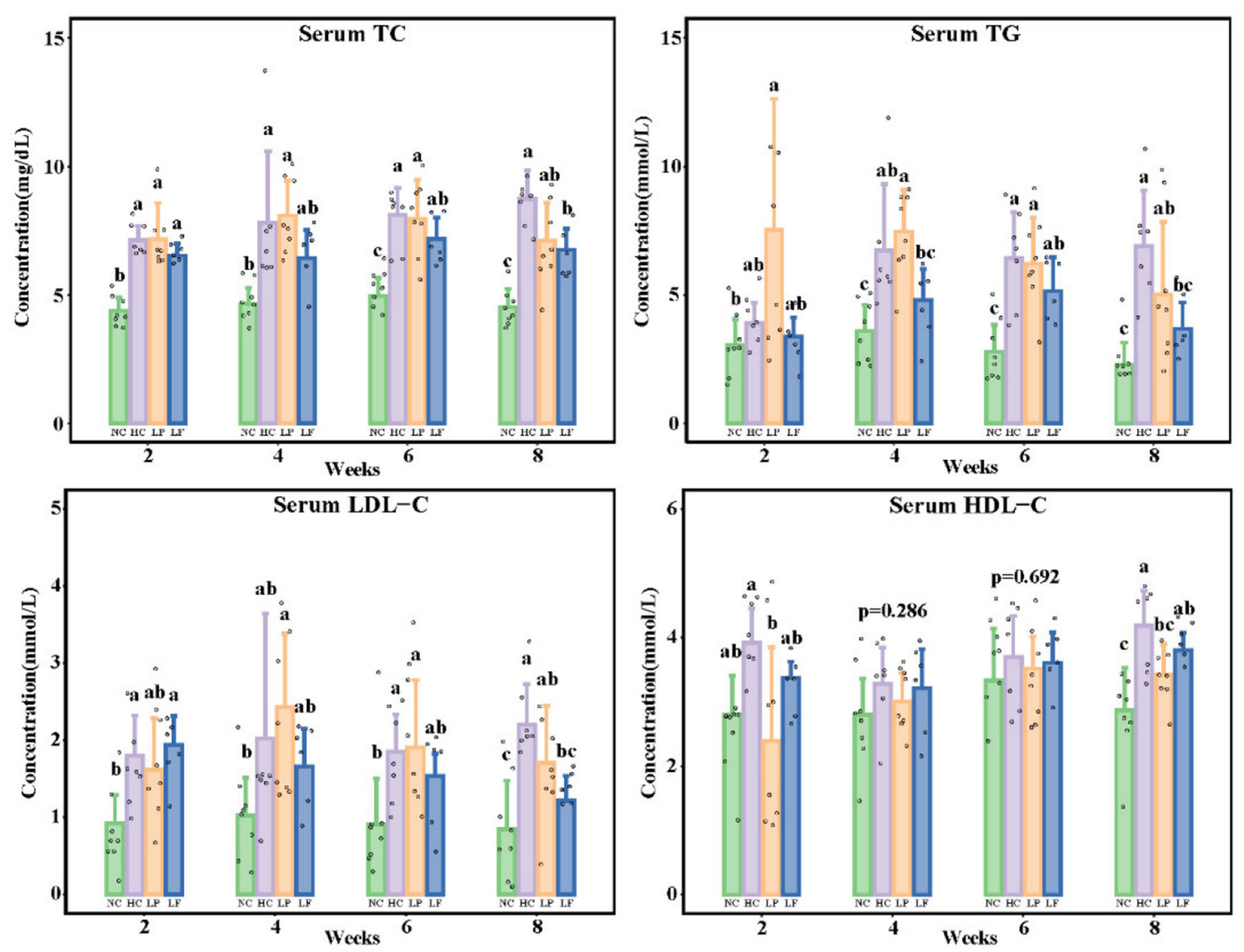

FIGURE 3 | Serum lipid levels of golden hamsters. Groups annotated with a, b, $c$ are significantly different with $P<0.05$ as determined by one-way ANOVA and a Tukey's test. TC, total cholesterol; TG, triglyceride; LDL-C, low-density lipoprotein cholesterol; HDL-C, high-density lipoprotein cholesterol; NC, negative control group; HC, positive control group; LP, Lactobacillus plantarum ZY08 supplemented group; LF, Lactobacillus fermentum ZJUIDS06 supplemented group. NC, HC, LP, and LF are colored in green, purple, orange, and blue, respectively.

Business Machines, Corp., Armonk, USA). Differences at $P$ $<0.05$ were considered significant. QIIME2 and R packages (v3.6.3) were used to analyze the sequencing data. The ASV table in QIIME2 was used to calculate the ASV-level alpha diversity indices, such as Chaol richness estimator, Observed species, Shannon diversity index, Simpson index, Faith's PD, Pielou's evenness, Good's coverage, and to visualize box plots. The richness and evenness of ASVs between the samples were compared by the generated ASV-level ranked abundance curves. Bray-Curtis metrics (57), non-metric multidimensional scaling (NMDS), and unweighted pair-group method with arithmetic means (UPGMA) hierarchical clustering (58) were applied to investigate the structural variation of microbial communities across samples in Beta diversity analysis. PERMANOVA (Permutational multivariate analysis of variance) (59) and ANOSIM (Analysis of similarities) $(60,61)$ in QIIME2 were applied to assess the significance of differentiation for the microbiota structure among groups. LEfSe (Linear discriminant analysis effect size) was applied to detect differentially abundant taxa around the groups in the default parameters (62). The correlation between the genus level abundance of cecum microbiota and SCFA was analyzed by Spearman's correlation coefficient and plotted using the R package "corrplot."

\section{RESULTS}

\section{Golden Hamster Body Weights and Daily Dietary Intake}

The body weight for each golden hamster was recorded weekly and the means for each group were calculated (Figure 2). In the pre-fed and early intervention periods, the mean values of body weight were comparable among all groups. However, the mean body weights for golden hamsters in the positive control group (HC), Lactobacillus plantarum supplemented group (LP), and Lactobacillus fermentum supplemented group (LF) were higher than those in the negative control group (NC) after 6 and 8 weeks of the experiment. Similar results for body weights were observed in the three groups fed the high cholesterol diet (HC, LP, and LF). There was no significant difference in the daily nutritional intake between all groups. 

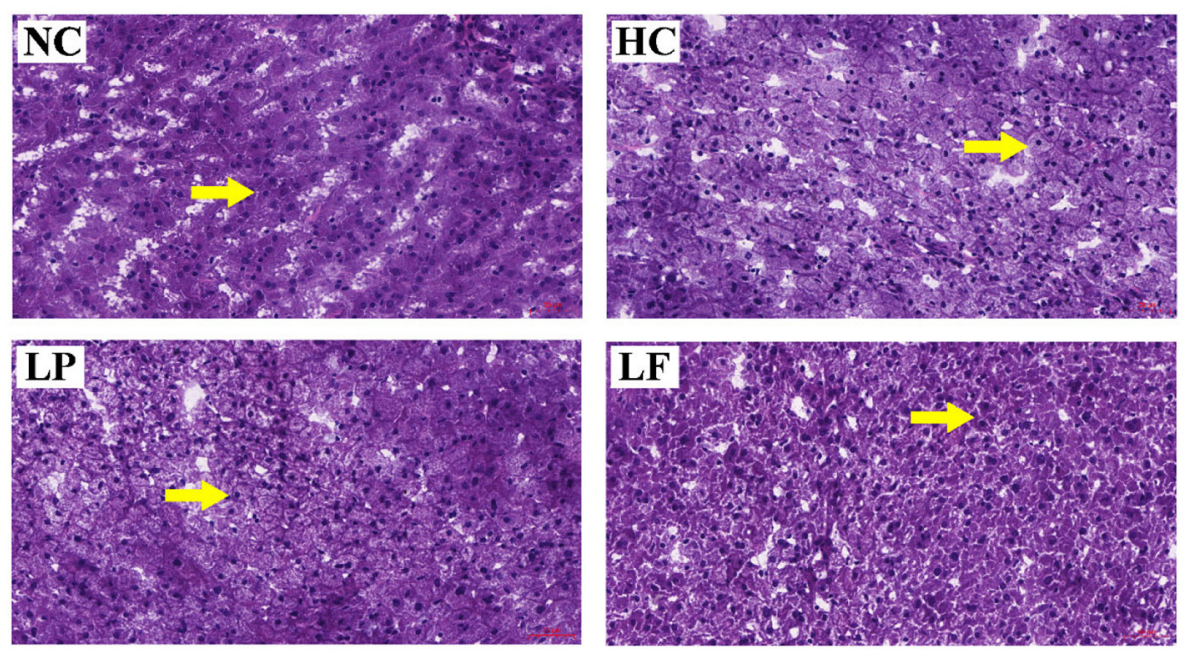

FIGURE 4 | Histological staining of liver tissues from hyperlipidemic golden hamsters after 8-weeks of treatment. Arrows indicate the situation of cytoplasm near the nucleus. Specimens were visualized and image captured using light microscopy (H \& E stain, magnification: $\times 200$, Scale bar, $50 \mu \mathrm{m})$. NC, negative control group; HC, positive control group; LP, Lactobacillus plantarum ZY08 supplemented group; LF, Lactobacillus fermentum ZJUIDS06 supplemented group.

\section{Effect of LAB on Serum Lipids}

Serum lipids, including TC, LDL-C, HDL-C, and TG were determined every 2 weeks. Exposing the golden hamsters to a high cholesterol diet for 2 weeks increased serum TC and LDL-C levels, indicating that the addition of $0.1 \%$ cholesterol and $10 \%$ lard to the feed was suitable for inducing hypercholesteremia. After 8 weeks, the serum TC, TG, HDL-C, and LDL-C levels were overall significantly different among all the groups (Figure 3). Ingestion of Lactobacillus fermentum ZJUIDS06 for 8 weeks reduced serum TC and TG levels in the golden hamsters fed the high cholesterol diet by $1.97 \mathrm{mg} / \mathrm{dL}$ and 3.21 $\mathrm{mmol} / \mathrm{L}$, respectively, and reduced serum LDL-C by $44.8 \%$, while Lactobacillus plantarum ZY08 did not have such an effect. Ingestion of Lactobacillus fermentation ZJUIDS06 did not affect the serum HDL-C levels in golden hamsters fed the high cholesterol diet, while ingestion of Lactobacillus plantarum ZY08 reduced the HDL-C levels.

\section{Liver Histology}

Histopathological analysis was performed on the harvested livers using hematoxylin-eosin staining to assess the effects of LAB supplementation on hepatocyte steatosis (Figure 4). The cytoplasm near the nucleus was compact in the hepatocytes of golden hamsters in the NC group. In the HC and LP group, a large number of lipid droplets appeared in the cytoplasm near the nucleus of the hepatocytes. Remarkably, ingestion of Lactobacillus fermentum ZJUIDS06 reduced the number of lipid droplets that were present in the cytoplasm near the nucleus of the hepatocytes.

\section{Effect of LAB on SCFA}

The concentrations of acetic acid, butyric acid, and propionic acid in colon contents of golden hamsters are presented in Figure 5. The amount of total SCFAs in the NC group was lower

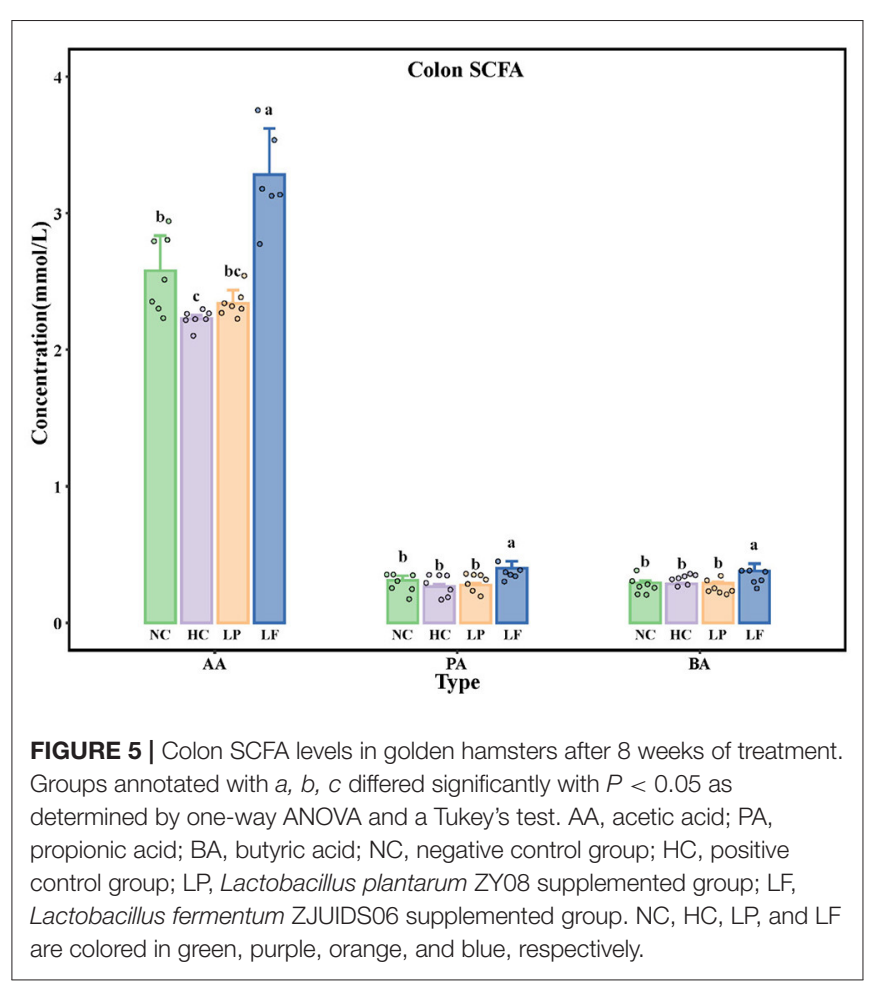

than that of the HC group. Golden hamsters supplemented with Lactobacillus fermentum ZJUIDS06 had higher levels of total SCFAs compared to any other group. Ingestion of Lactobacillus fermentum ZJUIDS06 administration for 8 weeks increased acetic acid, propionic acid, and butyric acid by $1.06,0.13$, and 0.10 $\mathrm{mmol} / \mathrm{L}$, respectively, while ingestion of Lactobacillus plantarum ZY08 did not have similar effects. 


\section{Effect of LAB on Tissue Weight}

Liver and the epididymal fat pad (EFP) are the two major adipose tissue depots in golden hamsters (63). Therefore, the liver and epididymal fat pad (EFP) weights were recorded to assess the effects of $\mathrm{LAB}$ on fat accumulation in the hamsters (Supplementary Table 2). The weights of these organs were comparable among the HC, LP, and LF groups. The liver and EFP weight of golden hamsters in the HC, LP, and LF groups were higher than those in the $\mathrm{NC}$ group.

\section{Effect of LAB on Cecum Microbiota}

To assess the effects of Lactobacillus fermentum ZJUIDS06 and Lactobacillus plantarum ZY08 intervention on the cecum microbiota of hyperlipidemic golden hamsters (Supplementary Figure 3), a MiSeq sequencing-based analysis of bacterial 16S rRNA (V3-V4 region) in cecum content was performed. After being spliced and optimized, 27 samples were delineated into 40,453 OTUs at a 95\% similarity level with distance-based OTUs and richness, and rarefaction and Shannon index analysis indicating that the sequencing depth covered rare new phylotypes and most of the diversity (Supplementary Figure 4).

The caecal microbiota community was first assessed by analyzing species richness, or the number of species in a community, and species diversity, which is the number of species and abundance of each species that live in a particular location (64) (Figure 6A). The observed species and Chao indices of the LF group were significantly higher than the other groups, indicating that the L. fermentum ZJUIDS06 intervention increased cecal microbiota richness. However, the Simpson and Shannon indices showed no differences between any of the two groups, indicating that the LAB intervention did not influence the diversity of cecal microbiota.

Next, a Multidimensional Scaling (NMDS) analysis was applied to visualize the differences in community structure between the groups. NMDS is similar to a Principal coordinates analysis (PCoA) analysis, but MNDS analysis is decomposed by dimensionality reduction, and the data structure is simplified, so that the distribution characteristics of the samples can be described using a specific distance scale (65). Here, NMDS analysis revealed a distinct clustering of microbiota composition between the standard diet group (NC) and the high cholesterol diet groups (HC, LP, LF) (Figure 6B). The significant separation between groups was confirmed using an unweighted pair-group method with arithmetic means (UPGMA) hierarchical clustering (Figure 6C) which showed the similarity between samples in the form of a hierarchical tree and the clustering effect by branch length. An analysis of similarities (ANOSIM) revealed that the overall microbiota structure differed significantly between groups (Supplementary Table 3), indicating that the two LAB interventions induced different shifts in the structure of the caecal microbiota community.

Finally, discriminant taxonomic markers were identified with linear discriminant analysis effect size (LEfSe) using the non-parametric factorial Kruskal-Wallis $\mathrm{H}$-test (Figure 7). The LEfSe analysis resulted in three parts: The abundance histogram showed the specific distribution of significantly enriched species in different groups of the samples (Figure 7A); The species classification cladogram showed the taxonomic hierarchical distribution of significantly enriched species from the phylum to the genus level for each group of the samples (Figure 7B); the distribution histogram displayed the LDA value (LDA $>2$ ) of significantly different species, which is used to identify the significantly enriched taxa for each group and the important species identified (Figure 7C). Based on the results of the LEfSe analysis, Firmicutes and Bacteroidetes were the dominant phyla in the cecum of all groups. The cecum microbiota of golden hamsters fed a high cholesterol diet was characterized by an increased Firmicutes-toBacteroidetes ratio (Figure 7A). Remarkably, at the genus level, Lactobacillus fermentum ZJUIDS06 administration increased the relative abundance of Parabacteroides, Flavonifractor, and Lactobacillus plantarum ZY08 increased that of Faecalibaculum, Ruminococcus, and Desulfovibrio.

\section{Correlation Analysis}

To demonstrate whether the identified biomarkers were correlated with serum lipid indexes or cecum SCFAs, we performed an association analysis using the $\mathrm{R}$ package "corrplot" (Figure 8). At the family level, six of 10 marker taxa in the NC group, Muribaculaceae, Tannerellaceae, Atopobiaceae, Bacteroidaceae, Porphyromonadaceae, and Clostridiaceae, showed negative correlations with serum lipid indexes (Figure 8A). Three of five marker taxa in the HC group, Lachnospiraceae, Anaplasmataceae, and Ruminococcaceae, showed positive correlations with serum lipid indexes. Only Desulfomicrobiaceae in the LP group showed a positive correlation with acetic acid with a Spearman's correlation coefficient of 0.504 . The classes significantly correlated with serum lipid reduction and Lactobacillaceae and Bifidobacteriaceae are shown in Figure 8B.

At the genus level, three of the six identified biomarker taxa from the NC group, Olsenella, Bacteroides, and Muribaculum, showed negative correlations with serum lipid indexes. Kineothrix, Dehalobacterium, and Wolbachia from the HC group, Flavonifractor from the LF group, and Desulfovibrio from the LP group showed significantly positive correlations with serum lipid indexes. Interestingly, only Parabacteroides was enriched in the LF group presenting a negative correlation with LDL-C, as well as a positive correlation with SCFA levels, with a Spearman's correlation coefficient of 0.430 for acetic acid, 0.534 for pentanoic acid, and 0.416 for butyric acid, respectively (Figure 8C). In addition, Lactobacillus fermentum ZJUIDS06 administration increased both the relative abundance of Parabacteroides (Figure 8D) and the SCFA concentration in the colon content (Figure 8A).

\section{DISCUSSION}

Ingestion of LAB did not affect body weights of hyperlipidemic golden hamsters, while daily supplementation with Lactobacillus fermentum ZJUIDS06, at the dosages of $10^{9}$ CFU per $100 \mathrm{~g}$ body weight for 8 weeks, significantly reduced LDL-C, TC, and TG levels in the hyperlipidemic golden hamsters. The lack of 


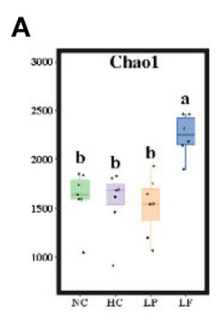

B
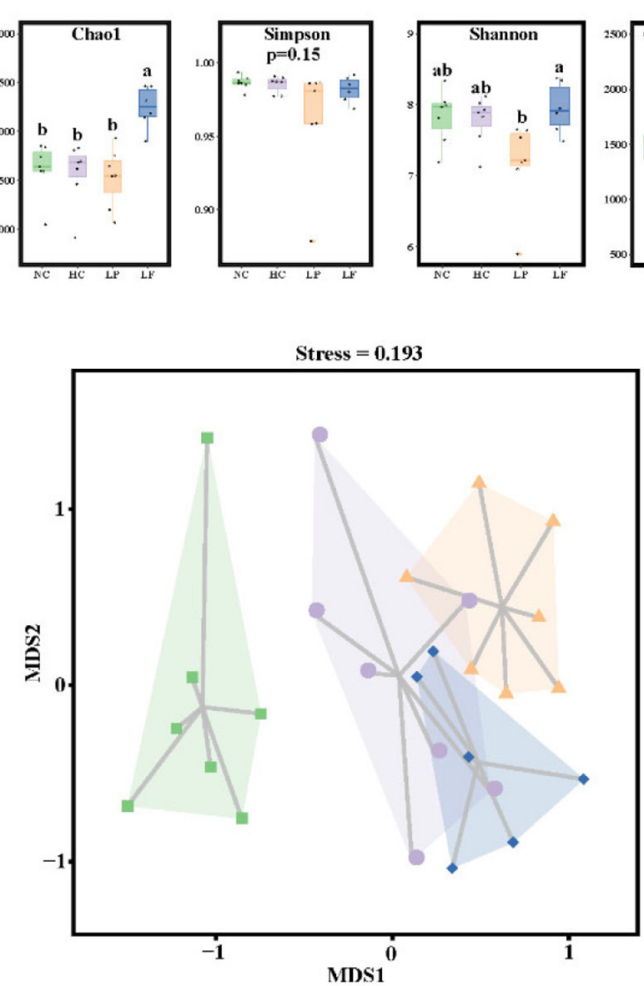

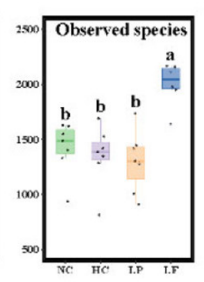

C

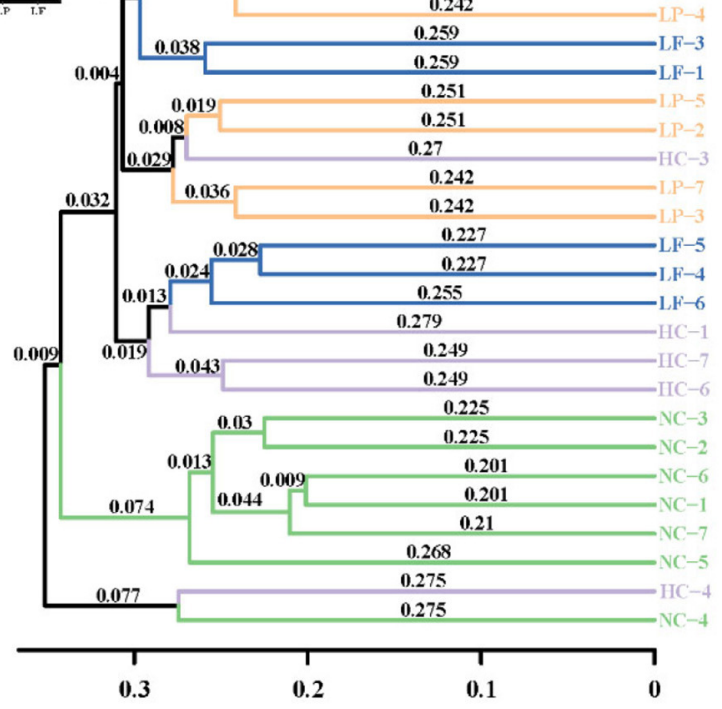

FIGURE 6 | (A) Box plot depicting alpha diversity in the experimental groups of golden hamsters. Groups annotated with $a, b$ significantly differed with $P<0.05$, respectively, as determined by a Kruskal-Wallis and Dunn's test. (B) NMDS based on Bray-Curtis distance. (C) Clustering tree depicting the samples clustering according to their similarity. NC, negative control group; HC, positive control group; LP, Lactobacillus plantarum ZY08 supplemented group; LF, Lactobacillus fermentum ZJUIDS06 supplemented group. NC, HC, LP, and LF are colored in green $(\boldsymbol{\square})$, purple $(\bullet)$, orange $(\mathbf{\Lambda})$, and blue $(\boldsymbol{\vee})$, respectively. a decreasing body weight effect following LAB administration in golden hamsters may be related to the feeding methods used. In previous studies where golden hamsters were exposed to unpredictable chronic mild stress or LAB administration, ingestion of probiotics significantly decreased the body weight gain (66-68). However, in the studies where feeding was unrestricted but monitored, probiotic intervention had no effect on body weight gain $(38,39,66,69)$, which was consistent with our findings here. Remarkably, Lactobacillus fermentum ZJUIDS06 exhibited cholesterol-lowering effect while Lactobacillus plantarum ZY08 did not have such effect. The variation in cholesterol-lowering effect between these two strains may relate to their different interactions with the host, and still needs to be further elucidated (70). The observation that the reduction of LDL-C, TC, and TG levels by Lactobacillus fermentum ZJUIDS06 in hyperlipidemic golden hamsters positively correlated with the levels of colon SCFAs, indicated that oral administration of Lactobacillus fermentum ZJUIDS06 may reduce serum lipids by inducing increased colon SCFAs. The effects of SCFAs on cholesterol metabolism in cellular models, hyperlipidemic animal models, and in human clinical trials have been well-documented (citation). Previously, butyrate was found to increase the activity of the liver $\mathrm{X}$ receptor $\mathrm{ABCG} 5$ and $\mathrm{G} 8$ expression and to decrease
NPC1L1 expression in Caco-2 cells (71). Concentration changes of SCFA indirectly activated ApoA-I expression with PPAR $\alpha$ transactivation, increased transcription of PPAR $\alpha$ and CPT1 and decreased transcription of KEAP1 in HepG2 cells (29). The dietary supply with SCFAs decreased serum lipids and promoted fecal excretion of bile acids in hyperlipidemic hamsters through up-regulation of SREBP2, LDLR, and CYP7A1 expression in the liver (28) and reduced the serum lipids in freshly weaned pigs by up-regulating the expression of hepatic FAS, CPT- $1 \alpha$, and SREBP-1 (72). Accordingly, the increase in colon SCFAs observed in this study may relate to the decrease in the serum lipids in hyperlipidemic golden hamsters. However, the role of SCFAs in mediating serum lipids still needs to be elucidated.

In previous reports, the cholesterol-lowering intervention strategies used in hyperlipidemic rodents increased the relative abundance of Lactobacillus or Bifidobacteria and decreased the ratio of Firmicutes to Bacteroides (39, 73-75). However, interventions used in this study did not have such effects (Figure 8B). The discrepancy between our research and other reports may relate to variations in the intestinal segments or animal models (41). Remarkably, in this research, Parabacteroides was the key symbiotic genus negatively correlated with LDL$\mathrm{C}$ and positively correlated with colon SCFAs. Species of Parabacteroides have been reported as symbiotic bacteria that 
A
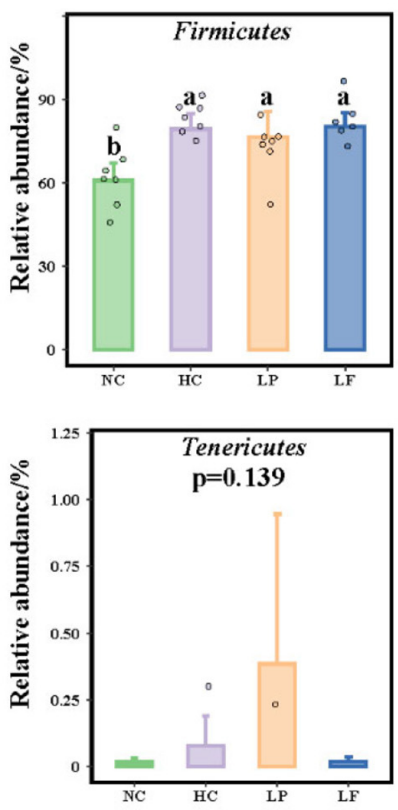
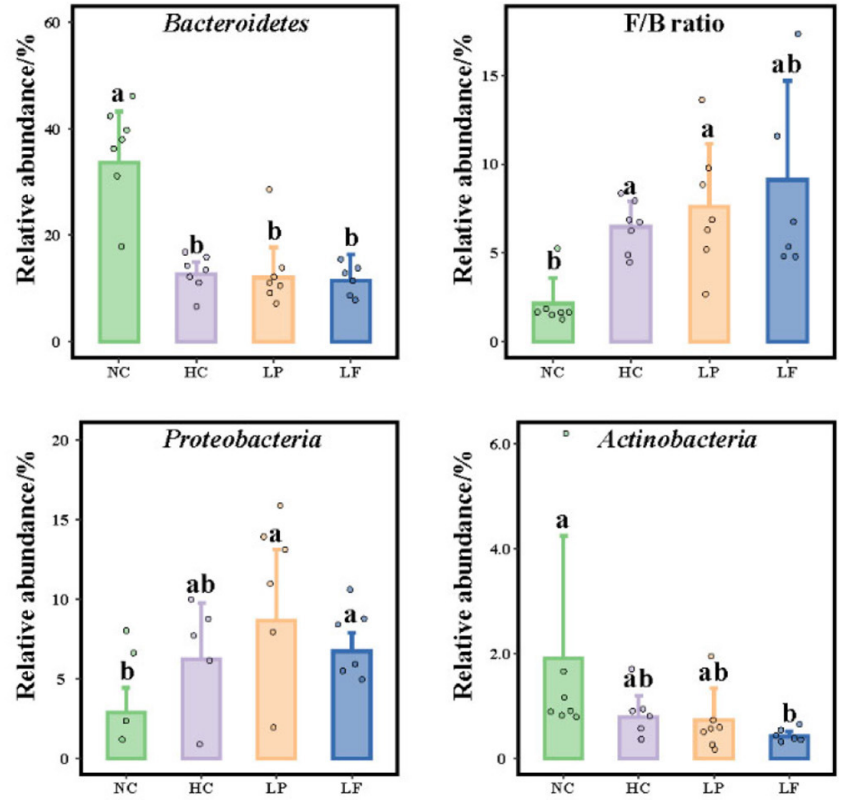

B

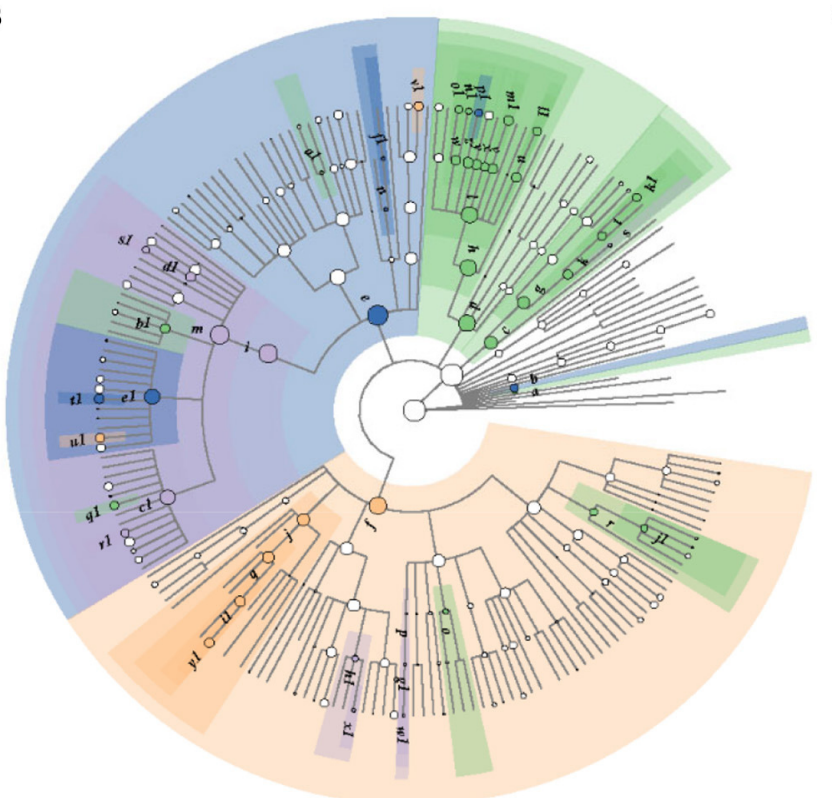

Taxa

a: $p$ Deferribacteres b: p_Ithubacter $c: p$ Actinobacteria d: $p$ Bacteroidetes e:p_Firmicutes f:p_Proteobacteria g:c Coriobacterila hic Bacteroidia i: $\mathrm{c}$ _Clostridia j:c Dettaproteobacteria k: o Coriobacteriales i:o- Bacterniales m: o Clostrialigles

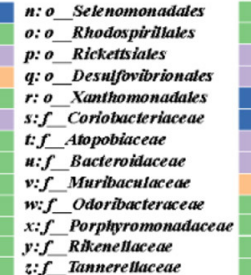

a1: $f$ Aerococcacese blif_Clostridiaceae c1:f Lachnospiraceate d1: $f$ Peptococcacear e1:f_Ruminococcaceae f1:5 Selenomonadaceae g1:f_Anaptasmataceae hI:f_oxalobacteraceae il: $f$ Desulfovibrionaceae j1if Xanthomonadaceae j1:f Xanthomo 11: g Bacteroides mi: $g$ Muribaculum

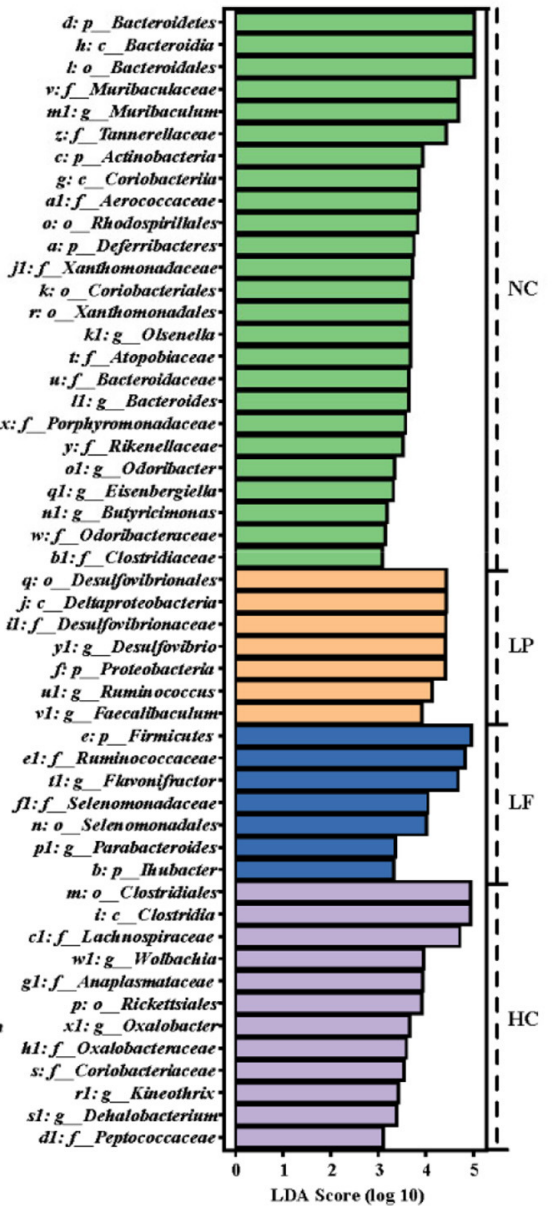

FIGURE 7 | (A) Caecum microbial composition of golden hamsters at the phylum level. (B) Taxa Lefse cladogram. (C) LDA Score (LDA >2). Groups annotated with letters $a, b$ were significantly different with $p<0.05$ as determined by a Kruskal-Wallis test and FDR correction. NC, negative control group; HC, positive control group; LP, Lactobacillus plantarum ZY08 supplemented group; LF, Lactobacillus fermentum ZJUIDS06 supplemented group. NC, HC, LP, and LF are colored in green, purple, orange, and blue, respectively. 
A

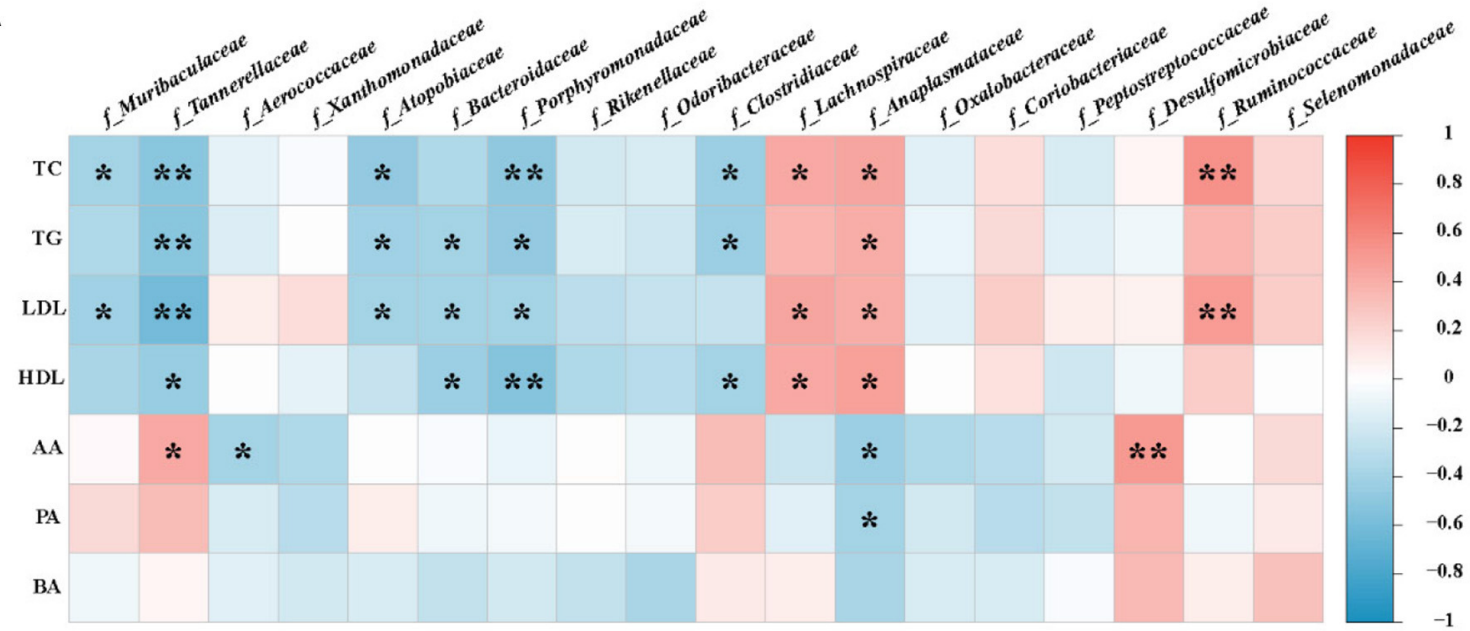

B

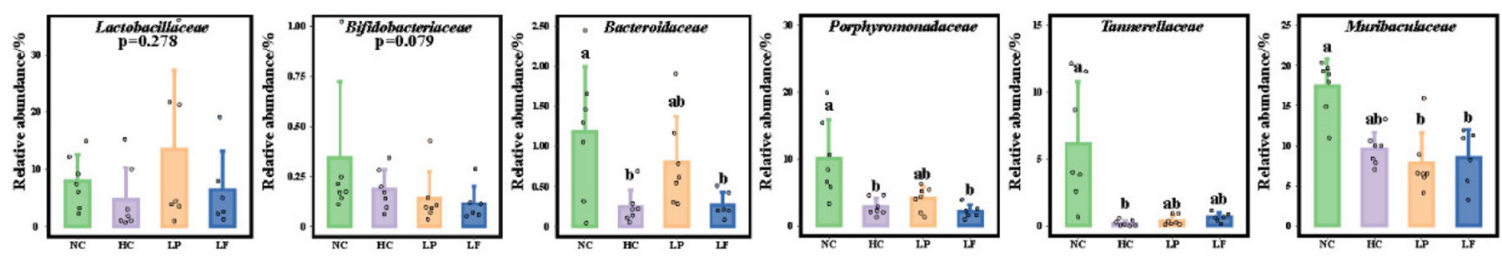

C

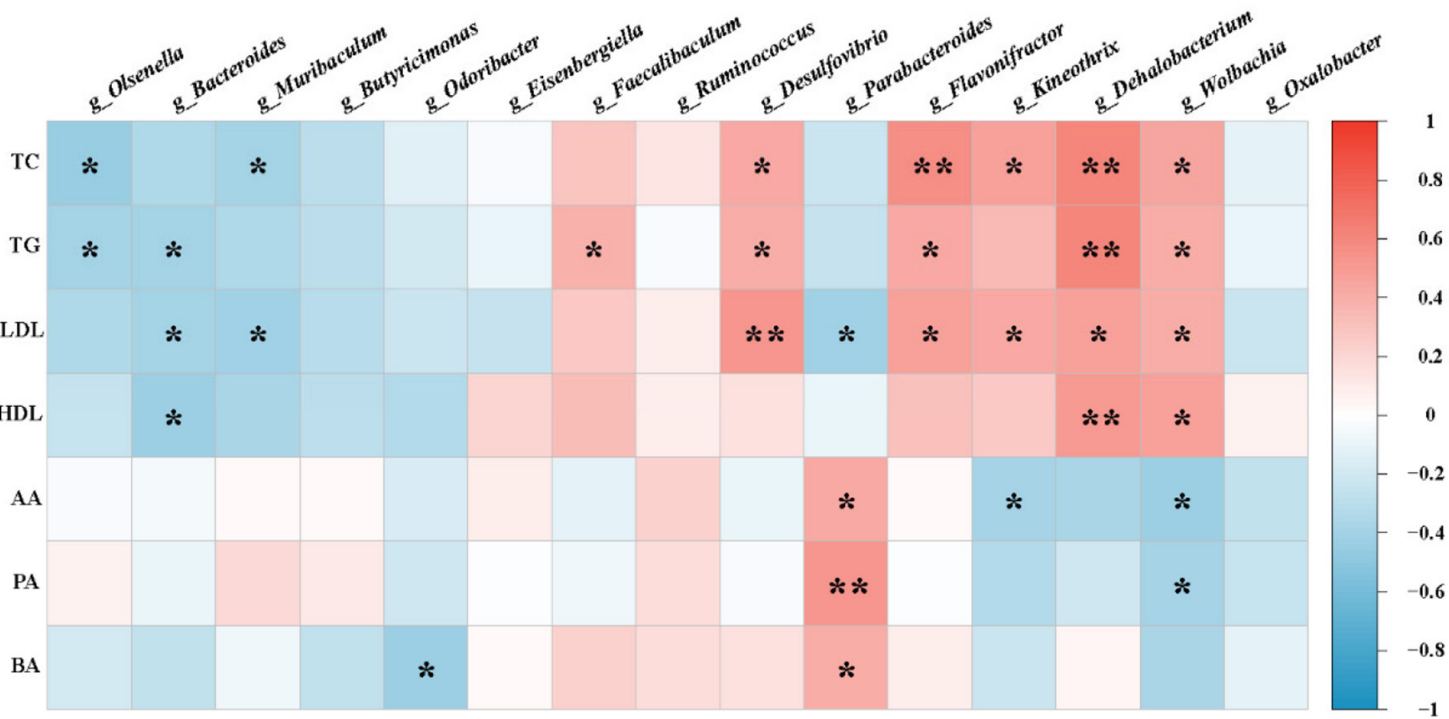

D

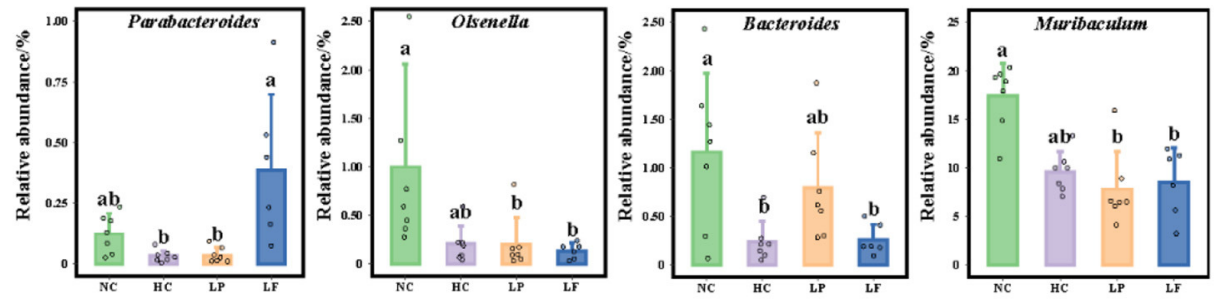

FIGURE 8 | (A) Heat map of Spearman's correlation coefficients between serum lipid indexes, colon SCFA, and marker taxa at the family level (f_bacteria). (B) The relative abundance of taxa at the family level that had a significantly negative correlation with the serum lipid indexes including Lactobacillaceae and Bifidobacteriaceae. 
FIGURE 8 | (C) Heat map of Spearman's correlation coefficient between serum lipid indexes, colon SCFA, and marker taxa at the genus level (g_bacteria). (D) The relative abundance of taxa at the genus level that had a significantly negative correlation with serum lipid indexes. Symbols * and ${ }^{* *}$ represent $P<0.05$ and $<0.01$, respectively. NC, negative control group; HC, positive control group; LP, Lactobacillus plantarum ZY08 supplemented group; LF, Lactobacillus fermentum ZJUIDS06 supplemented group; NC, HC, LP, and LF are colored in green, purple, orange, and blue, respectively. TC, serum total cholesterol; TG, serum triglyceride; LDL, serum LDL-C; HDL, serum HDL-C; AA, acetic acid in colon content; PA, propionic acid in colon content; BA, butyrate acid in colon content.

can alleviate obesity and metabolic dysfunction in mice (76). Parabacteroides goldsteinii relates to the anti-obesity effects of polysaccharides isolated from Hirsutella sinensis and water extract of Ganoderma lucidum mycelium in high-fat-diet (HFD) fed mice $(77,78)$. Oral treatment of HFD fed mice with live $P$. goldsteinii reduced obesity and was associated with increased adipose tissue thermogenesis. $P$. goldsteinii is a novel probiotic bacterium that may be used to treat obesity and associated metabolic disorders (78). These findings provide evidence that ingestion of Lactobacillus fermentum ZJUIDS06 may reduce serum lipids by enriching the commensal bacteria Parabacteroides.

Our results show that the oral administration of Lactobacillus fermentum ZJUIDS06 was not only positively correlated with Parabacteroides, but also with increased levels of SCFAs. The results of previous studies have found that some strains of Parabacteroides can produce SCFAs. For example, Parabacteroides acidifaciens sp. nov. ferments glucose into acetate acid, propionate, isobutyrate, and isopentanoate in vitro (79). However, the effects of Parabacteroides on in vivo SCFAs production remain inconclusive. Only one study found that oral administration of mice with alive Parabacteroides distanonis did not affect the level of acetate acid, propionate, isobutyrate, isopentanoate, and pentanoic acid in feces, but increased the level of jejunal succinic acid (76). Taken together, the correlation between Parabacteroides and in vivo SCFAs production still deserves further validation.

In conclusion, uptake of Lactobacillus fermentum ZJUIDS06 and Lactobacillus plantarum ZY08 did not prevent body weight gain in golden hamsters fed on a high cholesterol diet. However, oral administration of live Lactobacillus fermentum ZJUIDS06 in hyperlipidemic golden hamsters significantly increased colon SCFAs, and decreased the serum levels of LDL-C, TC, and TG, without affecting serum HDL-C, thus improving the colon SCFAs and serum lipid profiles. Both probiotics significantly altered the cecum microbiome, and the reduction of serum lipids following administration of Lactobacillus fermentum

\section{REFERENCES}

1. Stoekenbroek RM, Boekholdt SM, Fayyad R, Laskey R, Tikkanen MJ, Pedersen TR, et al. High-dose atorvastatin is superior to moderate-dose simvastatin in preventing peripheral arterial disease. Heart. (2015) 101:35662. doi: 10.1136/heartjnl-2014-306906

2. Pothineni NVK, Subramany S, Kuriakose K, Shirazi LF, Romeo F, Shah PK, et al. Infections, atherosclerosis, and coronary heart disease. Euro Heart J. (2017) 38:3195-201. doi: 10.1093/eurheartj/ehx362

3. Ni T, Fu Y, Zhou W, Chen M, Shao J, Zhou W, et al. Carotid plaques and neurological impairment in patients with acute cerebral infarction. PLoS ONE. (2020) 15:e0226961. doi: 10.1371/journal.pone.0226961
ZJUIDS06 was positively correlated with the relative abundance of Parabacteroides, which are commensal intestinal bacteria with probiotic characteristics. Our results give rise to a deeper understanding of the serum cholesterol-decreasing effects of certain probiotics.

\section{DATA AVAILABILITY STATEMENT}

The sequencing data was uploaded to the Sequence Read Archive (SRA) of NCBI and can be visited via accession number: PRJNA727412.

\section{ETHICS STATEMENT}

The animal study was reviewed and approved by the Animal Care and Use Committee of Zhejiang University.

\section{AUTHOR CONTRIBUTIONS}

DY, ZH, WL, and DR: research design. DY and JG performed in-vivo experiments. DY, JG, and ZZ collected the sample and data. DY and $\mathrm{ZH}$ analyzed the data. $\mathrm{ZH}$, JF, WW, WL, and DR revised the paper. All authors participated in the conception, design of the study, read, and approved the final manuscript.

\section{FUNDING}

This research work was supported by the Zheng Jiang province for Key Research \& Development Projects (Grant Number: 2019C02091).

\section{SUPPLEMENTARY MATERIAL}

The Supplementary Material for this article can be found online at: https://www.frontiersin.org/articles/10.3389/fnut.2021. 705763/full\#supplementary-material
4. Zhang Y, Wu N-Q, Li S, Zhu C-G, Guo Y-L, Qing P, et al. Non-HDL-C is a better predictor for the severity of coronary atherosclerosis compared with LDL-C. Heart Lung Circul. (2016) 25:975-81. doi: 10.1016/j.hlc.2016. 04.025

5. März W, Kleber ME, Scharnagl H, Speer T, Zewinger S, Ritsch A, et al. HDL cholesterol: reappraisal of its clinical relevance. Clin Res Cardiol. (2017) 106:663-75. doi: 10.1007/s00392-017-1106-1

6. Bellosta S, Corsini A. Statin drug interactions and related adverse reactions: an update. Expert Opinion Drug Safety. (2018) 17:25-37. doi: 10.1080/14740338.2018.1394455

7. Wong ND, Toth PP, Amsterdam EA. American Society for Preventive Cardiology. Most important advances in preventive cardiology during this 
past decade: viewpoint from the American Society for Preventive Cardiology. Trends Cardiovasc Med. (2021) 31:49-56. doi: 10.1016/j.tcm.2019.11.013

8. Kunwar S, Parekh JD, Chilukuri RS, Andukuri VA. Necrotizing autoimmune myopathy: a case report on statin induced rhabdomyolysis requiring immunosuppressive therapy. Drug Discov Ther. (2018) 12:315-7. doi: 10.5582/ddt.2018.01049

9. Özdemir IH, Copkiran Ö, Tikiz H, Tikiz C. Peripheral polyneuropathy in patients receiving long-term statin therapy. Turk Kardiyol Dern Ars. (2019) 47:554-63. doi: 10.5543/tkda.2019.78379

10. Paseban M, Butler AE, Sahebkar A. Mechanisms of statin-induced new-onset diabetes. J Cell Physiol. (2019) 234:12551-61. doi: 10.1002/jcp.28123

11. Liu $\mathrm{A}, \mathrm{Wu} \mathrm{Q}$, Guo J, Ares I, Rodríguez JL, Martínez-Larrañaga MR, et al. Statins: adverse reactions, oxidative stress and metabolic interactions. Pharmacol Ther. (2019) 195:54-84. doi: 10.1016/j.pharmthera.2018.10.004

12. Janssen L, Allard NaE, Saris CGJ, Keijer J, Hopman MTE, Timmers S. Muscle toxicity of drugs: when drugs turn physiology into pathophysiology. Physiol Rev. (2020) 100:633-72. doi: 10.1152/physrev.00002.2019

13. Hlivak P, Odraska J, Ferencik M, Ebringer L, Jahnova E, Mikes Z. One-year application of probiotic strain Enterococcus faecium M-74 decreases serum cholesterol levels. Bratisl Lek Listy. (2005) 106:67-72.

14. Nguyen TDT, Kang JH, Lee MS. Characterization of Lactobacillus plantarum PH04, a potential probiotic bacterium with cholesterol-lowering effects. Int $J$ Food Microbiol. (2007) 113:358-61. doi: 10.1016/j.ijfoodmicro.2006.08.015

15. Palaniyandi SA, Damodharan K, Suh JW, Yang SH. Probiotic Characterization of cholesterol-lowering Lactobacillus fermentum MJM60397. Probiotics Antimicrob Proteins. (2020) 12:1161-72. doi: 10.1007/s12602-019-09585-y

16. Yadav R, Khan SH, Mada SB, Meena S, Kapila R, Kapila S. Consumption of probiotic Lactobacillus fermentum MTCC: 5898 -fermented milk attenuates dyslipidemia, oxidative stress, and inflammation in male rats fed on cholesterol-enriched diet. Probiotics Antimicrobial Proteins. (2019) 11:50918. doi: 10.1007/s12602-018-9429-4

17. Jeon YB, Lee J-J, Chang HC. Characterization of juice fermented with EM and its cholesterol-lowering effects on rats fed a high-fat and high-cholesterol diet. Food Sci Nutr. (2019) 7:3622-34. doi: 10.1002/fsn3.1217

18. Aminlari L, Shekarforoush SS, Hosseinzadeh S, Nazifi S, Sajedianfard J, Eskandari MH. Effect of probiotics Bacillus coagulans and Lactobacillus plantarum on lipid profile and feces bacteria of rats fed cholesterol-enriched diet. Probiotics Antimicrobial Proteins. (2019) 11:1163-71. doi: 10.1007/s12602-018-9480-1

19. Kullisaar T, Zilmer K, Salum T, Rehema A, Zilmer M. The use of probiotic L. fermentum ME-3 containing Reg'Activ Cholesterol supplement for 4 weeks has a positive influence on blood lipoprotein profiles and inflammatory cytokines: an open-label preliminary study. Nutr J. (2016) 15:93. doi: 10.1186/s12937-016-0213-6

20. Tanaka Y, Hirose Y, Yamamoto Y, Yoshikai Y, Murosaki S. Daily intake of heat-killed Lactobacillus plantarum L-137 improves inflammation and lipid metabolism in overweight healthy adults: a randomized-controlled trial. Eur J Nutr. (2020) 59:2641-9. doi: 10.1007/s00394-019-02112-3

21. Park YE, Kim MS, Shim KW, Kim Y-I, Chu J, Kim B-K, et al. Effects of Q180 on postprandial lipid levels and intestinal environment: a doubleblind, randomized, placebo-controlled, parallel trial. Nutrients. (2020) 12:255. doi: 10.3390/nu12010255

22. Choi S-B, Lew L-C, Yeo S-K, Nair Parvathy S, Liong M-T. Probiotics and the BSH-related cholesterol lowering mechanism: a Jekyll and Hyde scenario. Crit Rev Biotechnol. (2015) 35:392-401. doi: 10.3109/07388551.2014.889077

23. Bustos AY, Font De Valdez G, Fadda S, Taranto MP. New insights into bacterial bile resistance mechanisms: the role of bile salt hydrolase and its impact on human health. Food Res Int. (2018) 112:25062. doi: 10.1016/j.foodres.2018.06.035

24. Jia E-T, Liu Z-Y, Pan M, Lu J-F, Ge Q-Y. Regulation of bile acid metabolismrelated signaling pathways by gut microbiota in diseases. J Zhejiang Univ Sci B. (2019) 20:781-92. doi: 10.1631/jzus.B1900073

25. Bosch M, Fuentes MC, Audivert S, Bonachera MA, Peiró S, Cuñé J. Lactobacillus plantarum CECT 7527, 7528 and 7529: probiotic candidates to reduce cholesterol levels. J Sci Food Agric. (2014) 94:803-9. doi: 10.1002/jsfa.6467

26. Lim FT, Lim SM, Ramasamy K. Pediococcus acidilactici LAB4 and Lactobacillus plantarum LAB12 assimilate cholesterol and modulate ABCA1,
CD36, NPC1L1 and SCARB1 in vitro. Beneficial Microbes. (2017) 8:97109. doi: 10.3920/BM2016.0048

27. Mishra AK, Kumar SS, Ghosh AR. Probiotic Enterococcus faecalis AG5 effectively assimilates cholesterol and produces fatty acids including propionate. FEMS Microbiol Lett. (2019) 366:fnz039. doi: 10.1093/femsle/fnz039

28. Zhao Y, Liu J, Hao $\mathrm{W}$, Zhu $\mathrm{H}$, Liang $\mathrm{N}$, He Z, et al. Structurespecific effects of short-chain fatty acids on plasma cholesterol concentration in male syrian hamsters. J Agric Food Chem. (2017) 65:10984-92. doi: 10.1021/acs.jafc.7b04666

29. Tayyeb JZ, Popeijus HE, Mensink RP, Konings MCJM, Mulders KHR, Plat $J$. The effects of short-chain fatty acids on the transcription and secretion of apolipoprotein A-I in human hepatocytes in vitro. J Cell Biochem. (2019) 120:17219-27. doi: 10.1002/jcb.28982

30. Zheng Y, Lu Y, Wang J, Yang L, Pan C, Huang Y. Probiotic properties of Lactobacillus strains isolated from Tibetan kefir grains. PLoS ONE. (2013) 8:e69868. doi: 10.1371/journal.pone.0069868

31. Bhat B, Bajaj BK. Multifarious cholesterol lowering potential of lactic acid bacteria equipped with desired probiotic functional attributes. 3 Biotech. (2020) 10:200. doi: 10.1007/s13205-020-02183-8

32. Psomas EI, Fletouris DJ, Litopoulou-Tzanetaki E, Tzanetakis N. Assimilation of cholesterol by yeast strains isolated from infant feces and Feta cheese. $J$ Dairy Sci. (2003) 86:3416-22. doi: 10.3168/jds.S0022-0302(03)73945-9

33. Sullivan MP, Cerda JJ, Robbins FL, Burgin CW, Beatty RJ. The gerbil, hamster, and guinea pig as rodent models for hyperlipidemia. Lab Anim Sci. (1993) 43:575-8.

34. Zhang Z, Wang H, Jiao R, Peng C, Wong YM, Yeung VSY, et al. Choosing hamsters but not rats as a model for studying plasma cholesterollowering activity of functional foods. Mol Nutr Food Res. (2009) 53:92130. doi: $10.1002 / \mathrm{mnfr} .200800517$

35. Guo CF, Yuan YH, Yue TL, Li JY. Hamsters are a better model system than rats for evaluating the hypocholesterolemic efficacy of potential probiotic strains. Mol Nutr Food Res. (2018) 62:e1800170. doi: 10.1002/mnfr.201800170

36. Dong B, Singh AB, Azhar S, Seidah NG, Liu J. High-fructose feeding promotes accelerated degradation of hepatic LDL receptor and hypercholesterolemia in hamsters via elevated circulating PCSK9 levels. Atherosclerosis. (2015) 239:364-74. doi: 10.1016/j.atherosclerosis.2015.01.013

37. Robins SJ, Fasulo JM, Patton GM, Schaefer EJ, Smith DE, Ordovas JM. Gender differences in the development of hyperlipemia and atherosclerosis in hybrid hamsters. Metabolism. (1995) 44:1326-31. doi: 10.1016/0026-0495(95)90038-1

38. Huang W-C, Chen Y-M, Kan N-W, Ho C-S, Wei L, Chan C$\mathrm{H}$, et al. Hypolipidemic effects and safety of Lactobacillus reuteri 263 in a hamster model of hyperlipidemia. Nutrients. (2015) 7:3767-82. doi: 10.3390/nu7053767

39. Sudun, L.iu S, Xiao C, Peng C, Liang L, He X, et al. Probiotic strains improve high-fat diet-induced hypercholesterolemia through modulating gut microbiota in ways different from atorvastatin. Food Funct. (2019) 10:6098109. doi: $10.1039 / \mathrm{C} 9 \mathrm{FO} 00444 \mathrm{~K}$

40. Fei Y, Wang Y, Pang Y, Wang W, Zhu D, Xie M, et al. Xylooligosaccharide modulates gut microbiota and alleviates colonic inflammation caused by high fat diet induced obesity. Front Physiol. (2019) 10:1601. doi: $10.3389 /$ fphys.2019.01601

41. Zmora N, Zilberman-Schapira G, Suez J, Mor U, Dori-Bachash M, Bashiardes $\mathrm{S}$, et al. Personalized gut mucosal colonization resistance to empiric probiotics is associated with unique host and microbiome features. Cell. (2018) 174:1388-405. doi: 10.1016/j.cell.2018.08.041

42. Liu JH, Zhang ML, Zhang RY, Zhu WY, Mao SY. Comparative studies of the composition of bacterial microbiota associated with the ruminal content, ruminal epithelium and in the faeces of lactating dairy cows. Microb Biotechnol. (2016) 9:257-68. doi: 10.1111/1751-7915.12345

43. Yu HJ, Jing C, Xiao N, Zang XM, Zhang CY, Zhang X, et al. Structural difference analysis of adult's intestinal flora basing on the 16S rDNA gene sequencing technology. Eur Rev Med Pharmacol Sci. (2020) 24:12983-92. doi: 10.26355/eurrev_202012_24203

44. Zeng Q, An S. Identifying the biogeographic patterns of rare and abundant bacterial communities using different primer sets on the loess plateau. Microorganisms. (2021) 9:139. doi: 10.3390/microorganisms9010139 
45. Bokulich NA, Kaehler BD, Rideout JR, Dillon M, Bolyen E, Knight $\mathrm{R}$, et al. Optimizing taxonomic classification of marker-gene amplicon sequences with QIIME 2's q2-feature-classifier plugin. Microbiome. (2018) 6:90. doi: 10.1186/s40168-018-0470-z

46. Martin M. Cutadapt removes adapter sequences from high-throughput sequencing reads. EMBnet J. (2011) 17:13. doi: 10.14806/ej.17.1.200

47. Callahan BJ, Mcmurdie PJ, Rosen MJ, Han AW, Johnson AJA, Holmes SP. DADA2: High-resolution sample inference from Illumina amplicon data. Nat Methods. (2016) 13:581-3. doi: 10.1038/nmeth.3869

48. Katoh K, Misawa K, Kuma, K-I, Miyata T. MAFFT: a novel method for rapid multiple sequence alignment based on fast Fourier transform. Nucl Acids Res. (2002) 30:3059-66. doi: 10.1093/nar/gkf436

49. Price MN, Dehal PS, Arkin AP. FastTree: computing large minimum evolution trees with profiles instead of a distance matrix. Mol Biol Evolut. (2009) 26:1641-50. doi: 10.1093/molbev/msp077

50. Chao A. Nonparametric estimation of the number of classes in a population. Scand J Stat. (1984) 11:265-70.

51. Shannon CE. A mathematical theory of communication. Bell Syst Tech J. (1948) 27:379-423. doi: 10.1002/j.1538-7305.1948.tb01338.x

52. Shannon CE. A mathematical theory of communication. Bell Syst Tech J. (1948) 27:623-56. doi: 10.1002/j.1538-7305.1948.tb00917.x

53. Simpson EH. Measurement of diversity. Nature. (1949) 163:688. doi: 10.1038/163688a0

54. Lozupone CA, Hamady M, Kelley ST, Knight R. Quantitative and qualitative $\beta$ diversity measures lead to different insights into factors that structure microbial communities. Appl Environ Microbiol. (2007) 73:157685. doi: 10.1128/AEM.01996-06

55. Lozupone C, Knight R. UniFrac: a new phylogenetic method for comparing microbial communities. Appl Environ Microbiol. (2005) 71:822835. doi: 10.1128/AEM.71.12.8228-8235.2005

56. Kõljalg U, Nilsson RH, Abarenkov K, Tedersoo L, Taylor AFS, Bahram M, et al. Towards a unified paradigm for sequence-based identification of fungi. $\mathrm{Mol}$ Ecol. (2013) 22:5271-7. doi: 10.1111/mec.12481

57. Bray JR, Curtis JT. An ordination of the upland forest communities of southern wisconsin. Ecol Monogr. (1957) 27:326-49. doi: 10.2307/1942268

58. Ramette A. Multivariate analyses in microbial ecology. FEMS Microbiol Ecol. (2007) 62:142-60. doi: 10.1111/j.1574-6941.2007.00375.x

59. Mcardle BH, Anderson MJ. Fitting multivariate models to community data: a comment on distance-based redundancy analysis. Ecology. (2001) 82:2907. doi: 10.1890/0012-9658(2001)082[0290:FMMTCD]2.0.CO;2

60. Clarke KR. Non-parametric multivariate analyses of changes in community structure. Austral Ecol. (1993) 18:11743. doi: 10.1111/j.1442-9993.1993.tb00438.x

61. Warton DI, Wright ST, Wang Y. Distance-based multivariate analyses confound location and dispersion effects. Methods Ecol Evolut. (2012) 3:89101. doi: 10.1111/j.2041-210X.2011.00127.x

62. Segata N, Izard J, Waldron L, Gevers D, Miropolsky L, Garrett WS, et al. Metagenomic biomarker discovery and explanation. GenomeBiology.com. (2011) 12:R60. doi: 10.1186/gb-2011-12-6-r60

63. Harris RBS. Denervation as a tool for testing sympathetic control of white adipose tissue. Physiol Behav. (2018) 190:3-10. doi: 10.1016/j.physbeh.2017.07.008

64. Pyron M. Characterizing communities. Nat Educ Knowl. (2010) 3:39.

65. Legendre P, Legendre L. Numerical Ecology: Second English Edition. Amsterdam: Elsevier Science BV (1998). p. 870.

66. Ting W-J, Kuo W-W, Hsieh DJ-Y, Yeh Y-L, Day C-H, Chen Y-H, et al. Heat killed Lactobacillus reuteri GMNL-263 reduces fibrosis effects on the liver and heart in high fat diet-hamsters via TGF- $\beta$ suppression. Int J Mol Sci. (2015) 16:25881-96. doi: 10.3390/ijms161025881

67. Hsieh F-C, Lan C-CE, Huang T-Y, Chen K-W, Chai C-Y, Chen W-T, et al. Heat-killed and live Lactobacillus reuteri GMNL-263 exhibit similar effects on improving metabolic functions in high-fat diet-induced obese rats. Food Funct. (2016) 7:2374-88. doi: 10.1039/C5FO01396H

68. Avolio E, Fazzari G, Zizza M, De Lorenzo A, Di Renzo L, Alò R, et al. Probiotics modify body weight together with anxiety states via proinflammatory factors in HFD-treated Syrian golden hamster. Behav Brain Res. (2019) 356:390-9. doi: 10.1016/j.bbr.2018.09.010

69. Bhathena J, Martoni C, Kulamarva A, Urbanska AM, Malhotra M, Prakash S. Orally delivered microencapsulated live probiotic formulation lowers serum lipids in hypercholesterolemic hamsters. J Med Food. (2009) 12:310 9. doi: 10.1089/jmf.2008.0166

70. Campana R, van Hemert S, Baffone W. Strain-specific probiotic properties of lactic acid bacteria and their interference with human intestinal pathogens invasion. Gut Pathog. (2017) 9:1-12. doi: 10.1186/s13099-017-0162-4

71. Chen Y, Xu C, Huang R, Song J, Li D, Xia M. Butyrate from pectin fermentation inhibits intestinal cholesterol absorption and attenuates atherosclerosis in apolipoprotein E-deficient mice. J Nutr Biochem. (2018) 56:175-82. doi: 10.1016/j.jnutbio.2018.02.011

72. Jiao AR, Diao H, Yu B, He J, Yu J, Zheng P, et al. Oral administration of short chain fatty acids could attenuate fat deposition of pigs. PLoS ONE. (2018) 13:e0196867. doi: 10.1371/journal.pone.0196867

73. Huang Y, Wang J, Cheng Y, Zheng Y. The hypocholesterolaemic effects of Lactobacillus acidophilus American type culture collection 4356 in rats are mediated by the down-regulation of Niemann-Pick C1-like 1. Br J Nutr. (2010) 104:807-12. doi: 10.1017/S0007114510001285

74. Xie N, Cui Y, Yin Y-N, Zhao X, Yang J-W, Wang Z-G, et al. Effects of two Lactobacillus strains on lipid metabolism and intestinal microflora in rats fed a high-cholesterol diet. BMC Complement Alternative Med. (2011) 11:53. doi: 10.1186/1472-6882-11-53

75. Liang T-T, Tong L-T, Geng D-H, Wang L-L, Zhou X-R, Pu H-Y, et al. Wheat Gluten regulates cholesterol metabolism by modulating gut microbiota in hamsters with hyperlipidemia. J Oleo Sci. (2019) 68:90922. doi: 10.5650/jos.ess 18257

76. Wang K, Liao M, Zhou N, Bao L, Ma K, Zheng Z, et al. Parabacteroides distasonis alleviates obesity and metabolic dysfunctions via production of succinate and secondary bile acids. Cell Rep. (2019) 26:222-35. doi: 10.1016/j.celrep.2018.12.028

77. Chang C-J, Lin C-S, Lu C-C, Martel J, Ko Y-F, Ojcius DM, et al. Ganoderma lucidum reduces obesity in mice by modulating the composition of the gut microbiota. Nat Commun. (2015) 6:7489. doi: 10.1038/ncomm s8489

78. Wu T-R, Lin C-S, Chang C-J, Lin T-L, Martel J, Ko Y-F, et al. Gut commensal plays a predominant role in the anti-obesity effects of polysaccharides isolated from. Gut. (2019) 68:248-62. doi: 10.1136/gutjnl-2017-315458

79. Wang Y-J, Xu X-J, Zhou N, Sun Y, Liu C, Liu S-J, et al. Parabacteroides acidifaciens sp. nov., isolated from human faeces. Int J Syst Evolut Microbiol. (2019) 69:761-6. doi: 10.1099/ijsem.0.0 03230

Conflict of Interest: WW was employed by Zhejiang YIMING food CO. LTD.

The remaining authors declare that the research was conducted in the absence of any commercial or financial relationships that could have led to a potential conflict of interest.

Copyright (C) 2021 Yang, Lyu, Hu, Gao, Zheng, Wang, Firrman and Ren. This is an open-access article distributed under the terms of the Creative Commons Attribution License (CC BY). The use, distribution or reproduction in other forums is permitted, provided the original author(s) and the copyright owner(s) are credited and that the original publication in this journal is cited, in accordance with accepted academic practice. No use, distribution or reproduction is permitted which does not comply with these terms. 